\title{
Heterodyne spectroscopy of polariton spinor interactions
}

\author{
N. Takemura, ${ }^{1, *}$ S. Trebaol,,${ }^{1}{ }^{\dagger}$ M. Wouters, ${ }^{2}$ M. T. Portella-Oberli, ${ }^{1}$ and B. Deveaud ${ }^{1}$ \\ ${ }^{1}$ Laboratory of Quantum Optoelectronics, École Polytechnique Fédérale de Lausanne, CH-1015 Lausanne, Switzerland \\ ${ }^{2}$ Theory of Quantum and Complex Systems, Universiteit Antwerpen, B-2610 Antwerpen, Belgium \\ (Received 16 October 2013; revised manuscript received 23 October 2014; published 14 November 2014)
}

\begin{abstract}
We report on spinor polariton interactions in GaAs based microcavities. This investigation is carried out by means of heterodyne polarized pump-probe spectroscopy. We show the dependence of the energy renormalization of the lower and upper polariton resonances with cavity detuning for different polariton densities. We use the exciton-photon based Gross-Pitaevskii equation to model the experiment for both lower and upper polariton modes. The theoretical results reproduce qualitatively the experimental observations revealing the magnitude and sign of the parallel and antiparallel spin interaction strength. We evidence the strong influence of the biexciton resonance on the antiparallel spin polariton energy shift and provide the exciton-biexciton coupling constant. We derive our results in the lower polariton basis using the Gross-Pitaevskii equation, from which we express analytically the spinor polariton interactions and identify the clear role of the biexciton resonance.
\end{abstract}

DOI: 10.1103/PhysRevB.90.195307

PACS number(s): 78.67.De, 71.36.+c

\section{INTRODUCTION}

Microcavity exciton polaritons are quasiparticles resulting from the strong coupling between excitons and photons [1]. Polaritons exhibit mutual interactions coming from their excitonic content and a light effective mass inherited from the photon. Collected photons emitted from the cavity allow reading out the polariton properties. As a matter of fact, a polariton fluid is an ideal tool for investigating quantum phenomena in solid-state systems. Polariton interactions in semiconductor microcavities play a crucial role in a wide variety of topics such as nonlinear optical effects [2-6], polariton superfluidity [7-10], and Bose Einstein condensation [11].

All these topics enlighten that polaritons provide a concrete realization of a many-body interacting system. Since polaritons carry a spin, spinor interactions characterize fundamental physical processes in polariton quantum systems. This results in anisotropic nonlinearities at the origin of many effects, such as: stimulated spin dynamics of polaritons $[12,13]$, transport of spin polarized polaritons [14], optical spin Hall effect [15-17], generation of polarization vortices [18,19] and half quantum vortices [20,21], spontaneous polarization buildup in Bose-Einstein condensation [22], bistability [23], multistability [24-26], and polariton switching [27,28].

Despite their importance, the spinor polariton interactions have been determined only indirectly. Several experiments indicate that the interaction of polaritons with antiparallel spins is attractive [29-31], while in others it appears to be repulsive [25-27,32]. Furthermore, theoretical works predict that spinor polariton interaction strengths depend on the cavity detuning [31,33-35]. It is very important to note that each investigation uses its own experimental condition: resonant or nonresonant excitation, cavity detuning, and time scale. This influences the measurement and the determination of the interaction constants. Furthermore, previous studies

\footnotetext{
*naotomo.takemura@epfl.ch

†Present address: UMR FOTON, CNRS, Université de Rennes 1, Enssat, F22305 Lannion, France.
}

concentrate on the lower polariton behavior, omitting the upper polariton branch.

In this paper, we report on the study of lower and upper polariton energy renormalization as a function of the polariton density and the exciton-photon cavity detuning in the semiconductor microcavity. To carry out this investigation, we focus on the lower and upper polariton resonances through spectrally resolved pump-probe spectroscopy. We employ pump-probe pulse co- and counter-circular polarization configurations in order to investigate polariton-polariton interactions with parallel and antiparallel spins respectively. The results reveal both the repulsive and attractive character of the spinor polariton interactions through the measured energy shift of the probed lower and upper polariton resonances. Furthermore, the amplitude and sign of the lower and upper polariton energy shifts as a function of the cavity detuning are determined for different polariton densities. Our theoretical model is developed in the framework of the spinor Gross-Pitaevskii equation in the exciton-photon basis. Comparison between numerical simulations and experimental measurements allows us to extract the microscopic spinor interaction constants and the strength of the exciton-biexciton coupling constant. The latter evidences the strong influence of the biexciton resonance on the antiparallel spin energy renormalization. Through basis transformation from exciton-photon to lower polariton, we derive analytical expressions for the spinor polariton interaction constants, usually referred to as $\alpha_{1}$ and $\alpha_{2}[36]$.

This paper is organized as follows: In Sec. II, we describe the sample and the pump-probe experiment. Section III reports on the experimental results, while Sec. IV is dedicated to the theoretical simulations using exciton-photon based Gross-Pitaevskii equations. In Sec. V, we identify analytically the spinor polariton interactions using the polariton basis Gross-Pitaevskii equation. We then give a general conclusion in Sec. VI.

\section{EXPERIMENTAL METHOD}

This study is performed with a high quality III-V GaAsbased microcavity [37]. A single $8 \mathrm{~nm} \mathrm{In}_{0.04} \mathrm{Ga}_{0.96}$ As quantum 
well is introduced between a pair of GaAs/AlAs distributed Bragg-reflectors. The exciton-cavity detuning energy $\delta$ can be adjusted by changing the position of the laser spot on the wedged sample. The Rabi splitting is $\Omega=3.26 \mathrm{meV}$ at $\delta=0$. We use a pump-probe setup with both pump and probe 125 femtosecond pulses from a Ti:Sapphire laser. The center energy of the laser spectrum (the linewidth is $14.6 \mathrm{meV}$ ) is set between lower and upper polaritons, therefore the laser intensity is fixed for all cavity detunings. The experiments are performed under resonant excitation and at $4 \mathrm{~K}$.

We employ a heterodyne pump-probe technique [38]. The laser beam is split into three: The pump and probe are frequency shifted with acousto-optic modulators (AOM) by $75 \mathrm{MHz}$ and $79 \mathrm{MHz}$, respectively, and focused on the sample. In transmission, the probe signal is directed into an AOM together with the reference beam. The AOM, driven at $79 \mathrm{MHz}$, produces two $\pi$-shifted detection channels in which the reference and probe signal overlap spectrally. The mixed beams are dispersed in a spectrometer and the two $\pi$-shifted interferograms are subtracted to recover the pump-probe signal. This heterodyne pump-probe technique allows us to study the polariton interactions close to degenerate beams configuration at $\mathrm{k}=0$ and dramatically increases the signal-to-noise ratio. This enables a precise measurement of small energy shifts in the probe spectrum. The experimental setup is described in detail in our previous paper [10].

The principle of the experiment is the following: We generate a spin-up lower and upper polariton population with a $\sigma^{+}$circularly polarized pump pulse. By varying the power of the pump pulse, we control the spin-up polariton population. A small amount of spin-up (-down) lower and upper polaritons are then injected with a weak $\sigma^{+}\left(\sigma^{-}\right)$circularly polarized probe pulse. We spectrally probe the energy of the lower and upper polariton resonance by measuring the transmission spectrum of the probe pulse.

The strength of the spinor polariton interaction is determined from the renormalized energy shift of the polariton resonance due to the presence of the pump polaritons. If the spinor polariton interaction is repulsive, it shows a blueshift, otherwise a redshift appears for attractive interaction. The experiments are performed at different cavity detunings to highlight the role of the excitonic content on the polaritonpolariton interactions.

\section{EXPERIMENTAL RESULTS}

In Fig. 1, we display the co- and counter-circular pumpprobe spectra measured at a $-1.5 \mathrm{meV}$ cavity detuning for pump photon density of $3.0 \times 10^{13}, 5.9 \times 10^{13}$, and $1.2 \times 10^{14}$ photons pulse $\mathrm{em}^{-1} \mathrm{~cm}^{-2}$ together with the reference spectrum. The pump-probe spectra clearly show a blueshift of both the lower and upper polariton resonances in co-circular configuration, while for the counter-circular measurements they show a redshift. These results also show that, as the polariton density is increased, the polariton resonance energy shift increases. In order to extract the energy shifts of the polariton resonance quantitatively, each spectrum is fitted with a Lorentzian function. The energy shift is determined through the difference between the reference and the pump-probe spectra at a fixed
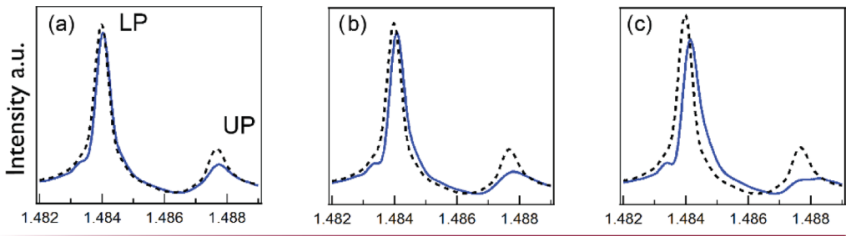

Pump Power $2 \mathrm{~mW}$
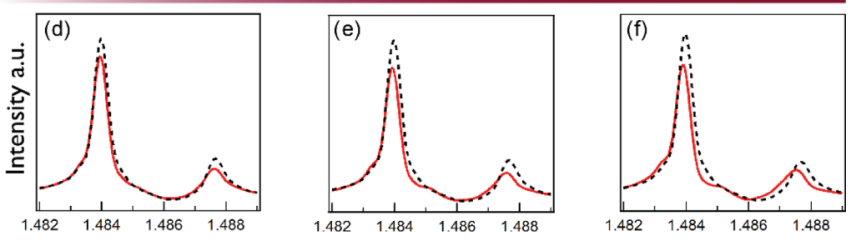

energy (eV)

energy (eV)

energy (eV)

FIG. 1. (Color online) Co- (blue) and counter- (red) circular polarization pump-probe spectra at $-1.5 \mathrm{meV}$ cavity detuning for different pump intensities: $3.0 \times 10^{13}(\mathrm{a}),(\mathrm{d}), 5.9 \times 10^{13}(\mathrm{~b}),(\mathrm{e})$, and $1.2 \times 10^{14}$ (c), (f) photons pulse $\mathrm{cm}^{-1}$. Transmitted probe spectra without pump (black dashed lines) and with pump (solid lines).

cavity detuning and for each pump power. We analyze both the lower and upper polariton energy shift for different polariton density and cavity detuning. We investigate separately the parallel and antiparallel spin polariton interactions.

\section{A. Parallel spin polariton interaction}

In Fig. 2(a), we show the dependence of the lower and upper polariton energy shifts on the cavity detuning, for different polariton densities. The results show that the blueshift for both resonances increases with polariton density and varies with cavity detuning. This evidences the repulsive interaction between polaritons with parallel spin for all cavity detunings. Notice the mirror behavior of the upper-lower polariton energy shift with cavity detuning. This reflects the role of the excitonic content of polariton states on polariton-polariton interactions. Indeed, for negative detuning the upper polariton being more excitoniclike than the lower polariton presents a larger blueshift. The reverse is true for positive detuning.

We would like to mention that the mirror symmetry axis is not at zero detuning but appears around $\delta=-0.8 \mathrm{meV}$. We might attribute this behavior to an onset of upper polariton scattering into an exciton reservoir. Therefore the upper polariton needs to be more excitonic to experience the same energy shift as the lower polariton.

\section{B. Antiparallel spin polariton interaction}

For the same set of polariton densities as above, we display the dependence of the upper and the lower polariton energy shifts with cavity detuning in Figs. 3(a) and 3(b), respectively. The lower and upper polariton resonances are redshifted, and this shift increases with polariton density. This result indicates the attractive interaction between polariton with antiparallel spin. The influence of the excitonic content of the polaritons for the interactions is again evidenced. Eventually, for lower polaritons, the energy shift increases from negative to positive cavity detunings. The reverse happens for the energy shift of the upper polaritons. 


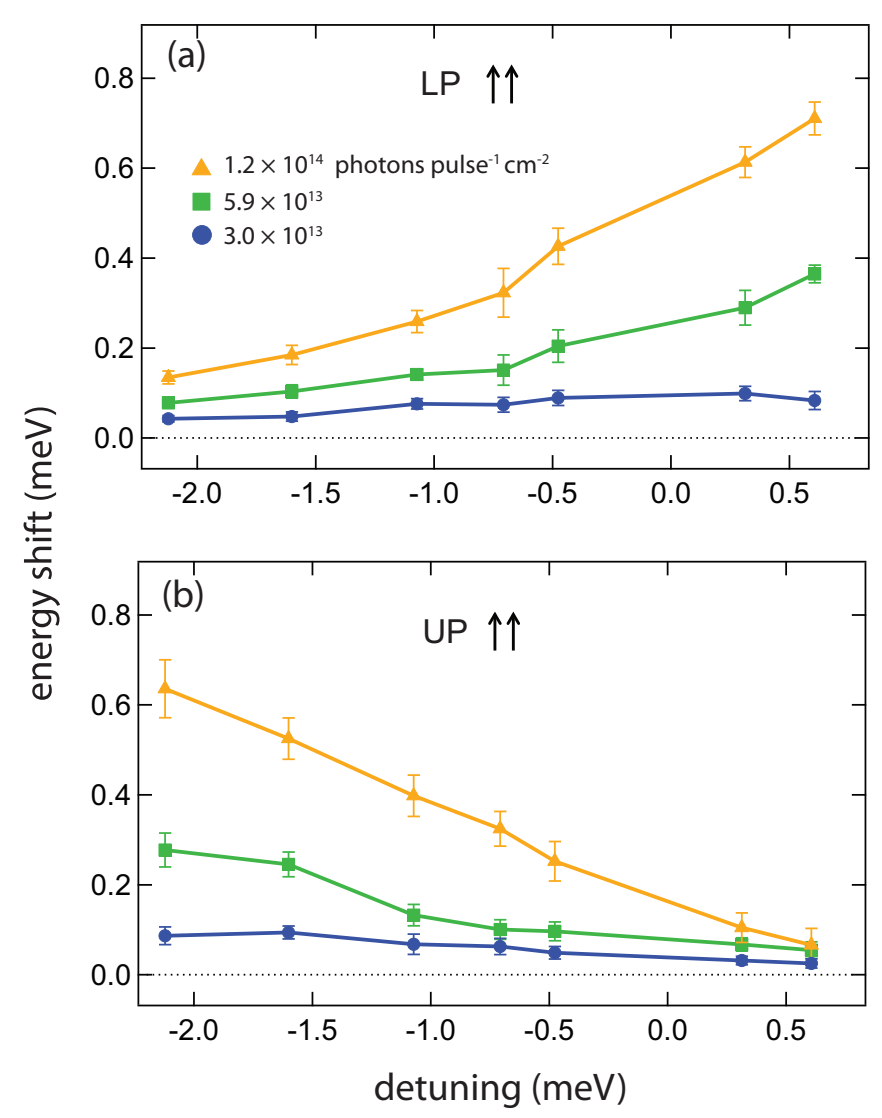

FIG. 2. (Color online) Energy shifts of lower (a) and upper (b) polariton resonances for co-circularly polarization configuration as a function of the cavity detuning. Three different symbols represent different pump intensities.

However, the dependence of the upper polariton energy shift on the cavity detuning is not a mirror image of the lower polariton. Here, the lower polaritons undergo a much stronger energy shift than the upper polaritons. Actually, the lower polariton energy renormalization is not only due to polariton-polariton interaction but also due to polaritonbiexciton coupling. It is important to note that for the lower polaritons from negative to positive cavity detuning, the energy of two lower polaritons approaches the energy of the biexciton resonance. This effect highlights the influence of the biexciton on the lower polariton energy renormalization for antiparallel spin polariton interactions. This result is in agreement with the polaritonic Feshbach resonance behavior recently observed [39], corroborating that biexcitons play a crucial role in the antiparallel spin polariton interactions.

\section{THEORETICAL MODEL}

Since our experimental approach consists of the excitation of both lower and upper polariton states in the nonlinear regime, cross interactions between polariton modes might appear leading to a cumbersome description of our results in the polariton basis. Therefore, we use the exciton-photonbased Gross-Pitaevskii equation to reproduce the experimental observations. Comparing the simulations to the experimental data will allow us to extract the spinor interaction constants

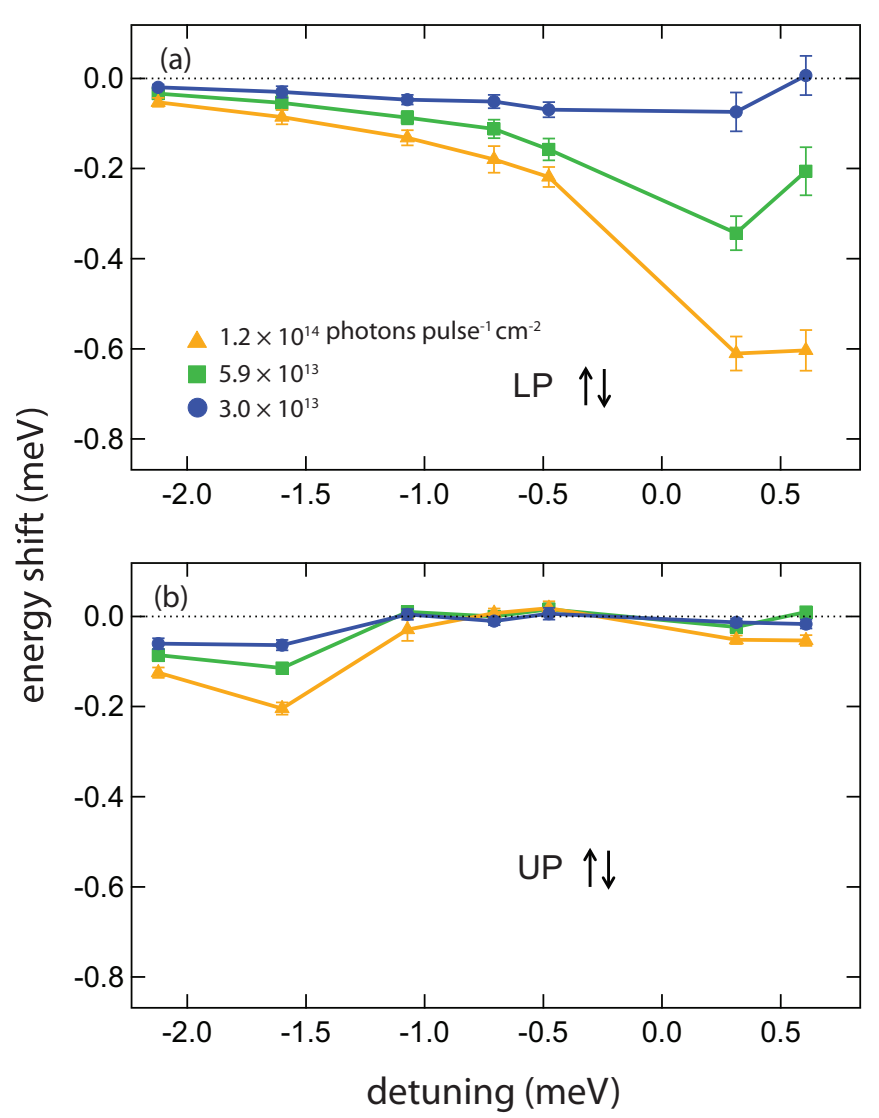

FIG. 3. (Color online) Energy shifts of lower (a) and upper (b) polariton resonances for counter-circularly polarization configuration as a function of the cavity detuning. Three different symbols represent different pump intensities.

in the exciton-photon basis Hamiltonian. In Sec. V, we will show how to transpose our analysis in the lower polariton basis yielding a comparison to previously reported results.

The exciton-photon basis Hamiltonian is the following:

$$
\hat{H}=\epsilon_{c} \hat{c}_{\uparrow}^{\dagger} \hat{c}_{\uparrow}+\epsilon_{x} \hat{x}_{\uparrow}^{\dagger} \hat{x}_{\uparrow}+\epsilon_{b} \hat{B}^{\dagger} \hat{B}+\Omega\left(\hat{c}_{\uparrow}^{\dagger} \hat{x}_{\uparrow}+\hat{x}_{\uparrow}^{\dagger} \hat{c}_{\uparrow}\right)+\hat{H}_{\text {int }} .
$$

The interacting part $\hat{H}_{\text {int }}$ is given by

$$
\begin{aligned}
\hat{H}_{\text {int }}= & \hat{H}_{\uparrow \uparrow}+\hat{H}_{\uparrow \downarrow}+\hat{H}_{b x} \\
= & g_{++} \hat{x}_{\uparrow}^{\dagger} \hat{x}_{\uparrow}^{\dagger} \hat{x}_{\uparrow} \hat{x}_{\uparrow}+g_{+-} \hat{x}_{\uparrow}^{\dagger} \hat{x}_{\downarrow}^{\dagger} \hat{x}_{\downarrow} \hat{x}_{\uparrow} \\
& +g_{b x}\left(\hat{B} \hat{x}_{\uparrow}^{\dagger} \hat{x}_{\downarrow}^{\dagger}+\hat{x}_{\uparrow} \hat{x}_{\downarrow} \hat{B}^{\dagger}\right) .
\end{aligned}
$$

The $\hat{c}, \hat{x}$, and $\hat{B}$ are, respectively, the photon, exciton and biexciton annihilation operators, while $\epsilon_{x}, \epsilon_{c}$, and $\epsilon_{B}$ are, respectively, the exciton, photon, and biexciton energy. The arrows $\uparrow$ and $\downarrow$ define the spin polarization as up and down. The $g_{++}$and $g_{+-}$are, respectively, the interaction strengths for parallel and antiparallel exciton interaction. The coupling between excitons and biexciton is given by $g_{b x}$. The same expressions hold when switching up and down spins in Eqs. (1) and (2). It is important to note that biexcitons can be created from two excitons with opposite spins via Coulomb interaction as described in Eq. (2). We call this the "bipolariton model" ( $x$ $x-B$ coupling) $[34,40]$. We could alternatively consider another 
type of biexciton creation mechanism: the "giant-oscillator strength model" ( $x-c-B$ coupling) $[41,42]$. In the latter, the biexciton has an oscillator strength and is formed through a photon absorption in the presence of an exciton. Although both models could be used, we choose the bipolariton model to describe our results, because, in our previous study performed on polariton Feshbach resonance [39], we found this model to be in better agreement with our experimental findings. However, for the sake of completeness, the giant-oscillator strength model is detailed in the Appendix.

It might be worth discussing the microscopic origin of these phenomenological interaction constants $g_{++}$and $g_{+-}$. To be precise, excitons are composite bosons composed of electron-hole pairs, thus exciton-exciton interactions are governed by the Coulomb interaction between electrons and holes. The simplest approach allowing us to calculate exciton-exciton interaction with parallel spins $\left(g_{++}\right)$is to compute the scattering matrix of excitons based on the Born approximation [43]. According to this calculation, in zero momentum scattering, which is the case for our experiment, the main contribution to the exciton-exciton interaction is electron (hole) exchange interaction. However, this calculation cannot explain the existence of the exciton interaction with antiparallel spins, because the exchange interaction disappears for the excitons with antiparallel spins [43]. Therefore, the inclusion of biexciton state ( $g_{b x}$ in our model) and the calculation beyond the Born approximation are important to explain the exciton interaction with antiparallel spin [44-47]. For example, such a calculation has been performed by Refs. $[45,46]$ through the summation of higher orders of scattering matrix including biexciton state. Their result shows first that the biexciton bound state introduces resonance scattering for exciton interactions with antiparallel spins. Second, the inclusion of the higherorder scattering matrices results in a strong modification of the exciton-exciton interaction both for parallel and antiparallel excitons: so-called "continuum correlations" [44-47]. These continuum correlations might be included in our phenomenological interaction constant with antiparallel spins $g_{+-}$, but the investigation of the exciton-exciton interaction starting from the electron-hole basis goes beyond the scope of our paper.

In our model, the phase space filling effect is omitted. Based on the result of Ref. [36], the exchange interaction of excitons with parallel spins $g_{++}^{\text {theor }}$ and the contribution from phase space filling $g_{\mathrm{PSF}}^{\text {theor }}$ can be estimated as $g_{++}^{\text {theor }}=3 a_{\mathrm{B}} e^{2} / \epsilon_{m} S$ (in the Born approximation) and $g_{\mathrm{PSF}}^{\text {theor }}=$ $\Omega / n_{\text {sat }} S$. Here $e$ and $\epsilon_{m}$, respectively, represent elementary charge and dielectric constant of the quantum wells. $S$ is a quantization area. Considering a 2D Bohr radius of GaAs $a_{\mathrm{B}}=6 \mathrm{~nm}$, a dielectric constant $\epsilon_{m}=13.9 \epsilon_{0} \quad\left(\epsilon_{0}\right.$ is the vacuum permittivity) [48], the Rabi splitting $\Omega=3.26 \mathrm{meV}$ and exciton saturation density $n_{\text {sat }} \simeq 7.5 \times 10^{11} \mathrm{~cm}^{-2}$, we obtain a ratio $g_{\mathrm{PSF}}^{\text {theor }} / g_{++}^{\text {theor }} \simeq 0.23$. This ratio indicates that the Coulomb term is the dominant contribution to the repulsive interaction of polaritons with parallel spins in our sample.

Using the Heisenberg equation of motion and mean field approximation [36], we obtain the equations of motion for the exciton, photon, and biexciton. In this Hamiltonian, biexcitons are created from spin-up and spin-down excitons, which indicates that counter-circular polarization configuration is mandatory to induce the biexciton effect $[34,40,49,50]$. The exciton $\left(\psi_{x}\right)$, photon $\left(\psi_{c}\right)$, and biexciton $\left(\psi_{B}\right)$ wave functions are described by the following exciton-photon Gross-Pitaevskii equation system:

$$
\begin{aligned}
i \hbar \dot{\psi}_{x, \uparrow}= & \left(\epsilon_{x}-i \frac{\gamma_{x}}{2}\right) \psi_{x, \uparrow}+\Omega \psi_{c, \uparrow}+2 g_{++}\left|\psi_{x, \uparrow}\right|^{2} \psi_{x, \uparrow} \\
& +g_{+-}\left|\psi_{x, \downarrow}\right|^{2} \psi_{x, \uparrow}+g_{b x} \psi_{B} \psi_{x, \downarrow}^{*} \\
i \hbar \dot{\psi}_{c, \uparrow}= & \left(\epsilon_{c}-i \frac{\gamma_{c}}{2}\right) \psi_{c, \uparrow}+\Omega \psi_{x, \uparrow}-F \\
i \hbar \dot{\psi}_{B}= & \left(\epsilon_{B}-i \frac{\gamma_{B}}{2}\right) \psi_{B}+g_{b x} \psi_{x, \uparrow} \psi_{x, \downarrow}
\end{aligned}
$$

Here, $\hbar$ is the reduced Planck constant. $\gamma_{x}, \gamma_{c}$, and $\gamma_{B}$ are, respectively, exciton, photon, and biexciton decay rate. In the numerical calculation, we use $\gamma_{x}=\gamma_{c}=0.53 \mathrm{meV}$. $F$ represents an external source of photons given by the laser pulses.

\section{A. Parallel spin polariton interaction}

In the co-circular polarization configuration, only the $g_{++}$ interaction term is involved. We omit the spin index since all polariton spins are the same. Our co-circular polarization pump-probe configuration is equivalent to that of $\chi^{(3)}$ parametric amplification. Thus, if we assume that the pump and probe beams have momentum $k_{p u}=0$ and $k_{p r}$, respectively, an idler beam appears with a momentum $k_{i}=2 k_{p u}-k_{p r}=-k_{p r}$. Since in the experiment $k_{p r} \simeq 0$, we neglect the dispersion of photons. We substitute the wave function:

$$
\psi_{x(c)}=\psi_{x(c)}^{p u}+\psi_{x(c)}^{p r} e^{i k x}+\psi_{x(c)}^{i} e^{-i k x}
$$

into the exciton-photon Gross-Pitaevskii equations (3a) and (3b). Assuming $\left|\psi_{x(c)}^{p u}\right| \gg\left|\psi_{x(c)}^{p r, i}\right|$ the feedback on the pump wave function from the signal and idler can be discarded, thus the pump wave function $\psi_{x(c)}^{p u}$ can be written as Eqs. (3a) and (3b). Additionally, we neglect the terms that do not conserve momentum. The dynamics of the set of wave functions $\vec{u}=\left(\psi_{x}^{p r}, \psi_{c}^{p r}, \psi_{x}^{i *}, \psi_{c}^{i *}\right)$ can be described as $i \hbar \dot{\vec{u}}=M^{++} \vec{u}-\vec{F}^{p r}$ [51], where $\vec{F}^{p r}$ represents the probe pulse excitation. The matrix $M^{++}$is given by

$$
M^{++}=\left(\begin{array}{cccc}
\epsilon_{x}+4 g_{++}\left|\psi_{x}^{p u}\right|^{2}-i \gamma_{x} / 2 & \Omega & 2 g_{++} \psi_{x}^{p u 2} & 0 \\
\Omega & \epsilon_{c}-i \gamma_{c} / 2 & 0 & 0 \\
-2 g_{++} \psi_{x}^{p u * 2} & 0 & -\left(\epsilon_{x}+4 g_{++}\left|\psi_{x}^{p u}\right|^{2}\right)-i \gamma_{x} / 2 & -\Omega \\
0 & 0 & -\Omega & -\epsilon_{c}-i \gamma_{c} / 2
\end{array}\right)
$$





FIG. 4. (Color online) Experimental lower (a) and upper (b) polariton energy shift as a function of the cavity detuning for parallel spin polaritons. Simulation of lower (c) and upper (d) polariton energy shift based on exciton-photon basis Hamiltonian. The blue and green line, respectively, represents the experimental (simulated) pump photon density: $3.0 \times 10^{13}\left(\left|F^{p u}\right|^{2}=1\right)$ and $5.9 \times 10^{13}\left(\left|F^{p u}\right|^{2}=2\right)$ photons pulse $^{-1} \mathrm{~cm}^{-2} \cdot\left|F^{p u}\right|^{2}$ is a normalized excitation pump photon density.

Since the lower and upper polaritons are excited with spectrally broad femtosecond pulses, we model the pump and probe photon pulses as instantaneous delta function pulses exciting the system simultaneously. By Fourier transforming the temporal photon probe wave functions, we obtain the spectra of the light emitted out of the cavity. Subtracting the pump-probe spectra from the reference spectra, we single out the energy shift of both lower and upper polariton resonances due to the presence of the pump pulse. The strength of our method to extract spinor interaction constants is based on the joint comparison of lower and upper polariton energy shifts.

In Figs. 4(a) and 4(b), we plot, respectively, the experimental data of lower and upper polariton energy shifts as a function of the cavity detuning, for pump photon densities of $3.0 \times 10^{13}$ and $5.9 \times 10^{13}$ photons pulse ${ }^{-1} \mathrm{~cm}^{-2}$. We obtain the parallel spin interaction constant $g_{++}=1 \mathrm{meV} / n_{0}$ by simulating the lower and upper polariton energy shifts for the two pump powers [Figs. 4(c) and 4(d)]. Here, $n_{0}$ is a normalization particle density for the excitation pump photon density $\left|F^{p u}\right|^{2}=1$. The experimental results are well reproduced: The lower polaritons show an increase of the energy shift from negative to positive cavity detuning, while the upper polaritons energy shift decreases. In fact, the lower polariton becomes more excitonic and the upper polariton becomes more photonic for increasing cavity detuning. This highlights the role of the excitonic content in polariton interactions.

\section{B. Antiparallel spin polariton interaction}

In the counter-circular polarization configuration, the large population of spin-up polaritons is probed with a small population of spin-down polaritons. Unlike the co-circular polarization configuration, the idler beam does not appear since the spin momentum conservation is not satisfied [52].

The dynamics of the spin-down probe can be described using three coupled equations of motion: $i \hbar \dot{\vec{u}}=M^{+-} \vec{u}-\vec{F}^{p r}$, where the vector $\vec{u}=\left(\psi_{x \downarrow}^{p r}, \psi_{c \downarrow}^{p r}, \psi_{B}\right)$ is composed of the probe exciton, probe photon, and biexciton wave functions respectively. The matrix $M^{+-}$is given by

$$
M^{+-}=\left(\begin{array}{ccc}
\epsilon_{x}+g_{+-}\left|\psi_{x \uparrow}^{p u}\right|^{2}-i \gamma_{x} / 2 & \Omega & g_{b x} \psi_{x \uparrow}^{p u *} \\
\Omega & \epsilon_{c}-i \gamma_{c} / 2 & 0 \\
g_{b x} \psi_{x \uparrow}^{p u} & 0 & \epsilon_{B}-i \gamma_{B} / 2
\end{array}\right) \text {. }
$$

In the matrix, $\psi_{x(c) \uparrow}^{p u}$ represents pump exciton (photon) wave function and follows the equations of motion Eqs. (3a) and ( $3 b)$. In the simulation, we set $\epsilon_{B}=2 \epsilon_{x}-2.5 \mathrm{meV}$ and $\gamma_{B}=1.1 \mathrm{meV}$. Similar to the co-circular simulation, we obtain 

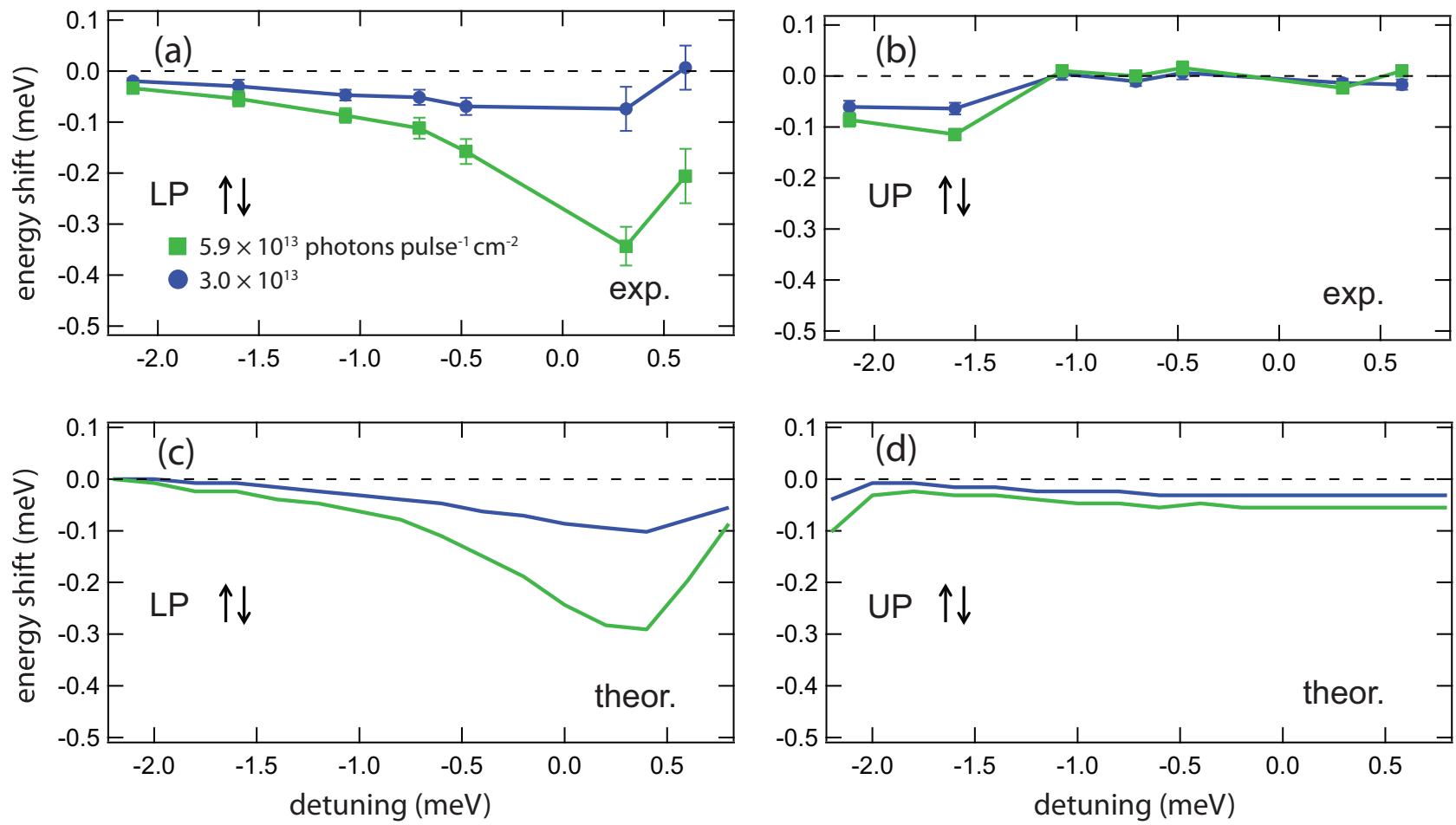

FIG. 5. (Color online) Experimental lower (a) and upper (b) polariton energy shifts as a function of the cavity detuning for antiparallel spin polaritons. Simulation of lower (c) and upper (d) polariton energy shift based on exciton-photon basis Hamiltonian. The blue and green line, respectively, represents the experimental (simulated) pump photon density: $3.0 \times 10^{13}\left(\left|F^{p u}\right|^{2}=1\right)$ and $5.9 \times 10^{13}\left(\left|F^{p u}\right|^{2}=2\right)$ photons pulse $\mathrm{cm}^{-1}$.

the interaction constants $g_{b x}=1.2 \mathrm{meV} / \sqrt{n_{0}}$ and $g_{+-}=$ $-1.2 \mathrm{meV} / n_{0}$, from the comparison between simulation and experiment of the lower and upper polariton energy shifts at $3.0 \times 10^{13}$ and $5.9 \times 10^{13}$ photons pulse ${ }^{-1} \mathrm{~cm}^{-2}$ pump photon densities. We plot in Fig. 5 the experimental and simulated lower and upper polariton energy shifts as a function of the cavity detuning. Similar to the experimental results, the simulation shows the enhancement of the lower polariton redshift energy from negative to positive cavity detuning [see Fig. 5 (c)]. This enhancement of the redshift of the lower polariton resonance comes from the scattering of lower polaritons (LP) via the biexciton state (BX) (LP-LP $\rightarrow$ BX). This effect plays an important role concerning the antiparallel spin polariton interactions and should be considered in the analysis of the renormalization of the lower polariton energy. Notice that the polaritonic Feshbach biexciton resonance [39] is analogous to the Feshbach resonance in cold atoms $[53,54]$ where the atom-atom interaction is altered due to a molecular bound state.

Concerning the upper polariton mode, the very small energy shift experimentally measured is well reproduced by the numerical simulation [Fig. 5(d)]. This behavior is specific of the counter-polarized configuration and then might be related to the presence of biexcitons. However, further investigations should be performed to deeply understand these observations. In summary, our approach is more general than previously reported studies since we consider a simultaneous excitation of lower and upper polariton branches. We probe the energy shifts of both branches and, through comparison between the numerical and experimental results, we estimate the interaction constants of the exciton-photon-biexciton Hamiltonian Eq. (2) as $g_{++}: g_{+-}: g_{b x} \simeq 1 / n_{0}:-1.2 / n_{0}: 1.2 / \sqrt{n_{0}}$ (meV). This ratio shows that the spinor polariton interaction with antiparallel spins, which is usually considered to be very weak, is comparable to that of parallel spins. However it is very important to note that, due to the commutation relation, a factor of 2 appears in front of $g_{++}$[Eq. (3a)]. As a consequence, the energy shift for parallel spins is twice the energy shift for antiparallel ones for the same density and cavity detuning. Additionally, the strong lower polariton energy shift with cavity detuning dependency appears due to the biexcitonexciton coupling. The joint study of lower and upper spinor polariton interaction described in this report is well reproduced in the exciton-photon basis.

Usually, in resonant excitation experiments, only the lower polariton branch is excited. Then, instead of the excitonic interaction constants $g_{++}, g_{+-}$, and $g_{b x}$, the parallel $\alpha_{1}$ and antiparallel $\alpha_{2}$ polariton-polariton interaction constants are defined within the lower-polariton basis. In order to validate and compare our results to other studies on polariton interactions, we describe below our observation in the lower polariton basis by changing the working basis from excitonphoton-biexciton to lower polariton basis.

\section{LOWER-POLARITON INTERACTION CONSTANTS}

When experiments with lower polaritons are considered, a continuous wave laser excitation is usually involved. In order to apply our extracted parameters to this configuration, we 
need to transform the exciton-photon basis Hamiltonian into lower-polariton Hamiltonian.

First, we rewrite the Hamiltonian Eq. (1) using the lowerpolariton basis defined as $\hat{p}_{\mathrm{lp}}=X \hat{x}+C \hat{c}$ and neglect terms that includes the upper polaritons [36]:

$$
\begin{aligned}
\hat{H}= & \epsilon_{c} \hat{c}_{\uparrow}^{\dagger} \hat{c}_{\uparrow}+\epsilon_{x} \hat{x}_{\uparrow}^{\dagger} \hat{x}_{\uparrow}+\Omega\left(\hat{c}_{\uparrow}^{\dagger} \hat{x}_{\uparrow}+\hat{x}_{\uparrow}^{\dagger} \hat{c}_{\uparrow}\right)+\hat{H}_{\mathrm{int}} \\
\simeq & \epsilon_{l p} \hat{p}_{l p}^{\dagger} \hat{p}_{l p}+g_{++}|X|^{4} \hat{p}_{l p, \uparrow}^{\dagger} \hat{p}_{l p, \uparrow}^{\dagger} \hat{p}_{l p, \uparrow} \hat{p}_{l p, \uparrow} \\
& +g_{+-}|X|^{4} \hat{p}_{l p, \uparrow}^{\dagger} \hat{p}_{l p, \downarrow}^{\dagger} \hat{p}_{l p, \downarrow} \hat{p}_{l p, \uparrow} \\
& +g_{b x}\left(X^{2} \hat{p}_{l p, \uparrow} \hat{p}_{l p, \downarrow} \hat{B}^{\dagger}+X^{* 2} \hat{p}_{l p, \uparrow}^{\dagger} \hat{p}_{l p, \downarrow}^{\dagger} \hat{B}\right) .
\end{aligned}
$$

Here $X$ and $C$ are Hopfield coefficients defined as

$$
|X|^{2}=\frac{1}{2}\left(1+\frac{\delta}{\sqrt{\delta^{2}+\Omega^{2}}}\right)
$$

and

$$
|C|^{2}=\frac{1}{2}\left(1-\frac{\delta}{\sqrt{\delta^{2}+\Omega^{2}}}\right) .
$$

The lower polariton energy $\epsilon_{l p}$ is calculated as

$$
\epsilon_{l p}=\frac{\epsilon_{c}+\epsilon_{x}}{2}-\frac{1}{2} \sqrt{\left(\epsilon_{c}-\epsilon_{x}\right)^{2}+\Omega^{2}} .
$$

Based on the Hamiltonian Eq. (7), we calculate the Heisenberg equations of motion applying mean-field approximation:

$$
\begin{aligned}
i \hbar \dot{\psi}_{l p, \uparrow}= & \left(\epsilon_{l p}-i \frac{\gamma_{l p}}{2}\right) \psi_{l p, \uparrow}+2 g_{++}|X|^{4}\left|\psi_{l p, \uparrow}\right|^{2} \psi_{l p, \uparrow} \\
& +g_{+-}|X|^{4}\left|\psi_{l p, \downarrow}\right|^{2} \psi_{l p, \uparrow}+g_{b x} X^{* 2} \psi_{l p, \downarrow}^{*} \psi_{B}-F,
\end{aligned}
$$

$$
i \hbar \dot{\psi}_{B}=\left(\epsilon_{B}-i \frac{\gamma_{B}}{2}\right) \psi_{B}+g_{b x} X^{2} \psi_{l p, \uparrow} \psi_{l p, \downarrow},
$$

where $\gamma_{l p}=|C|^{2} \gamma_{c}+|\mathrm{X}|^{2} \gamma_{\mathrm{x}}$ is the lower polariton decay rate. In the following, we address Eqs. (11) and (12) in parallel (A) and antiparallel (B) spin polariton frameworks.

\section{A. Parallel spin polariton interaction}

For the parallel spin configuration, the dynamics of the $\psi_{l p}$ wave function is given by

$$
i \hbar \dot{\psi}_{l p}=\left(\epsilon_{l p}-i \frac{\gamma_{l p}}{2}\right) \psi_{l p}+2 g_{++}|X|^{4}\left|\psi_{l p}\right|^{2} \psi_{l p}-F .
$$

Thus, the mean-field energy shift can be expressed as

$$
\Delta E_{++}=\alpha_{1}\left|\psi_{l p}\right|^{2},
$$

where $\left|\psi_{l p}\right|^{2}$ is the lower polariton density. The lower polariton interaction with parallel spins is thus defined as

$$
\alpha_{1}=2 g_{++}|X|^{4} \text {. }
$$

In Fig. 6(a), we plot the energy shift of the lower polariton for parallel spin interaction Eq. (14) using the value of $g_{++}$ extracted previously and pump polariton density $\left|\psi_{l p}\right|^{2}=0.2$. The energy shift follows a dependence given by the excitonic content of the lower polariton through the term $|X|^{4}$, as reported by Ciuti et al. [36].
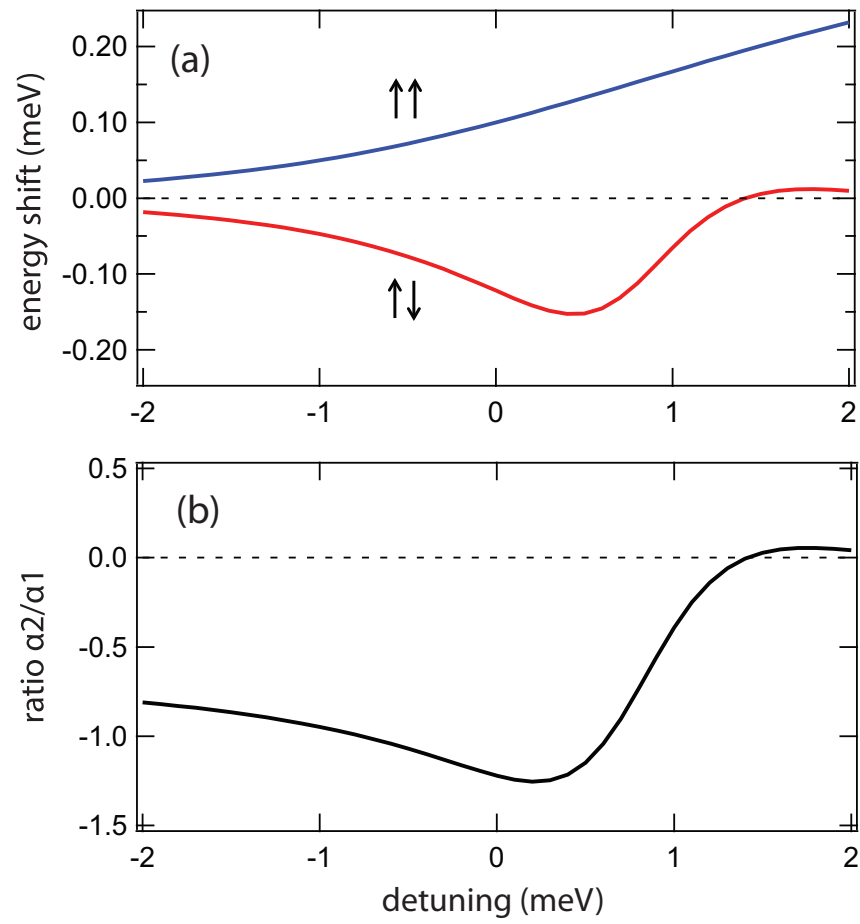

FIG. 6. (Color online) (a) The energy shift $\Delta E_{++}$(blue) and $\Delta E_{+-}$(red) obtained in Eqs. (14) and (19) as a function of the cavity detuning for the pump polariton density $\left|\psi_{l p, \uparrow}\right|^{2}=0.2$. (b) The ratio $\alpha_{2} / \alpha_{1}$ as a function of the cavity detuning.

\section{B. Antiparallel spin polariton interaction}

In order to obtain the lower polariton interaction with antiparallel spins, we consider pump-probe spectroscopy in the counter-circular polarization configuration. Assuming that the spin-up pump pulse is much stronger than the spin-down probe pulse, we can express the probe lower polariton dynamics as $i \hbar \dot{\vec{u}}=M \vec{u}-\vec{F}^{p r}$, where the vector $\vec{u}$ is $\vec{u}=\left(\psi_{l p \downarrow}^{p r}, \psi_{B}\right)$ and $\vec{F}^{p r}$ is a source term. The matrix $M$ is

$$
M=\left(\begin{array}{cc}
\epsilon_{l p}+g_{+-}|X|^{4}\left|\psi_{l p \uparrow}^{p u}\right|^{2}-i \gamma_{l p} / 2 & g_{b x} X^{* 2} \psi_{l p \uparrow}^{p u *} \\
g_{b x} X^{2} \psi_{l p \uparrow}^{p u} & \epsilon_{B}-i \gamma_{B} / 2
\end{array}\right) .
$$

Let us consider that the pump is resonant with the lowerpolariton energy, $\psi_{l p \uparrow}^{p u}=\left|\psi_{l p \uparrow}^{p u}\right| e^{-i \epsilon_{l p} t / \hbar}$, and probe with energy $\epsilon, \vec{F}^{p r}=(1,0) e^{-i \epsilon t / \hbar}$. For the steady state solutions, we assume that the pump wave function has a form $\psi_{l p \downarrow}^{p u}=$ $\psi_{l p \downarrow}^{p u}(\epsilon) e^{-i \epsilon_{l p} t / \hbar}$ and the biexciton wave function is expressed as $\psi_{B}=\psi_{B}(\epsilon) e^{-i\left(\epsilon+\epsilon_{l p}\right) t / \hbar}$. Replacing $\psi_{l p \uparrow}^{p u}$ and $\psi_{l p \uparrow}^{p u *}$ with $\left|\psi_{l p \uparrow}^{p u}\right|$ in the matrix $M$, we can obtain the steady-state solution of the probe lower polariton spectrum as

$$
\psi_{l p \downarrow}^{p r}(\epsilon)=\left(\begin{array}{ll}
1 & 0
\end{array}\right)\left[M-\left(\begin{array}{cc}
\epsilon & 0 \\
0 & \epsilon_{l p}+\epsilon
\end{array}\right)\right]^{-1}\left(\begin{array}{l}
1 \\
0
\end{array}\right) .
$$

The analytic solution of $\psi_{l p \downarrow}^{p r}(\epsilon)$ is

$$
\begin{aligned}
\psi_{l p \downarrow}^{p r}(\epsilon)= & {\left[\epsilon_{l p}-\epsilon+g_{+-}|X|^{4}\left|\psi_{l p \uparrow}^{p u}\right|^{2}-i \gamma_{l p} / 2\right.} \\
& \left.-\frac{g_{b x}^{2}|X|^{4}\left|\psi_{l p \uparrow}^{p u}\right|^{2}}{\epsilon_{B}-\epsilon_{l p}-\epsilon-i \gamma_{B} / 2}\right]^{-1} .
\end{aligned}
$$


Under the assumption of a weak exciton-biexciton coupling and a low pump excitation density $\left(g_{b x}\left|\psi_{l p \uparrow}^{p u}\right|<\gamma_{B} / 2\right)$, the two-mode solutions of $\psi_{l p \downarrow}^{p r}(\epsilon)$ might be approximated by a single solution. Indeed, substituting $\epsilon=\epsilon_{l p}$ and taking the real part of Eq. (A6), the pump induced energy shift $\Delta E_{+-}$ can be approximated as

$$
\Delta E_{+-} \simeq g_{+-}|X|^{4}\left|\psi_{l p \uparrow}^{p u}\right|^{2}-\frac{g_{b x}^{2}|X|^{4}\left|\psi_{l p \uparrow}^{p u}\right|^{2}\left(\epsilon_{B}-2 \epsilon_{l p}\right)}{\left(\epsilon_{B}-2 \epsilon_{l p}\right)^{2}+\left(\gamma_{B} / 2\right)^{2}}
$$

and the lower polariton interaction with antiparallel spins is defined as

$$
\alpha_{2} \simeq g_{+-}|X|^{4}-\frac{g_{b x}^{2}|X|^{4}\left(\epsilon_{B}-2 \epsilon_{l p}\right)}{\left(\epsilon_{B}-2 \epsilon_{l p}\right)^{2}+\left(\gamma_{B} / 2\right)^{2}} .
$$

This result shows that the two contributions to the energy shift in the counter-polarized configurations bring strong similarity to derived expressions for optical Feshbach resonance in cold atoms [55]. First, the term proportional to the excitonic content $|X|^{4}$ related to antiparallel spin polariton interaction is usually called background interaction term. Second, the lower-polariton-biexciton coupling term takes the form of a Lorentzian profile. Figure 6(a) displays the behavior of the lower-polariton energy shift in the counter-polarized configuration considering the value of $g_{+-}$and $g_{b x}$ extracted from the numerical simulations and pump polariton density $\left|\psi_{l p, \uparrow}\right|^{2}=0.2$. The energy shift displays a change in sign and amplitude when the energy of two lower polaritons crosses the energy of the biexciton, which here occurs at $\delta \approx 1 \mathrm{meV}$ [39].

In Fig. 6(b), we plot the ratio between counter- and co-polarized interactions. We obtain a ratio similar to the one reported previously using a different approach [31]. For negative detuning the ratio takes a negative value, -0.8 , that progressively increases until reaching -1.2 , then drops suddenly to a slightly positive value. This comparison to previous results strengthens the interest and generality of the method presented here.

\section{CONCLUSION}

In conclusion, we provided a direct demonstration that the lower and upper polaritons with parallel spins interact repulsively and attractively when possessing antiparallel spins. In addition, by modeling the experiments with the excitonphoton-based Gross-Pitaevskii equation, we extracted the spinor microscopic exciton interaction strengths. The joint analysis of the lower and upper polariton energy renormalization with cavity detuning reveals the dominant role of the biexciton in antiparallel spin configuration. Through the lower polariton basis model, we validate our results and in addition, we give the analytic expression for the polariton interaction with parallel spin $\alpha_{1}$ and with antiparallel spin $\alpha_{2}$ with the explicit role of the biexcitonic resonance effect.

\section{ACKNOWLEDGMENTS}

We thank Daniel Oberli for a fruitfull discussion and Mitchell D. Anderson for a critical reading of the manuscript. The present work is supported by the Swiss National Science Foundation under project $\mathrm{N}^{\circ} 135003$, the Quantum Photonics National Center of Competence in Research $\mathrm{N}^{\circ} 115509$, and from European Research Council under project Polaritonics contract $\mathrm{N}^{\circ} 219120$. The POLATOM Network is also acknowledged.

\section{APPENDIX: GIANT OSCILLATOR STRENGTH MODEL OF BIEXCITON}

\section{Exciton photon basis}

As stated in the paper, two different types of biexciton creation mechanism are possible: the bipolariton model $(x-x-$ $B$ ) $[34,40]$ and giant oscillator strength model $(x-c-B)[41,42]$. In the bipolariton model, the biexciton is formed through the Coulomb interaction between two excitons with opposite spins, while in the giant oscillator strength model, the biexciton is created through the absorption of a photon by a sea of excitons due to the oscillator strength of biexciton. In the paper we showed that the bipolariton model was able to reproduce accurately the observed features; here we will show that, similarly, the giant oscillator strength model gives a reasonable agreement with the experiment. In this model, the interaction Hamiltonian can be written as

$$
\begin{aligned}
\hat{H}_{\text {int }}= & \hat{H}_{\uparrow \uparrow}+\hat{H}_{\uparrow \downarrow}+\hat{H}_{b x}, \\
= & g_{++} \hat{x}_{\uparrow}^{\dagger} \hat{x}_{\uparrow}^{\dagger} \hat{x}_{\uparrow} \hat{x}_{\uparrow}+g_{+-} \hat{x}_{\uparrow}^{\dagger} \hat{x}_{\downarrow}^{\dagger} \hat{x}_{\downarrow} \hat{x}_{\uparrow} \\
& +g_{\Omega}\left(\hat{B}^{\dagger} \hat{x}_{\uparrow} \hat{c}_{\downarrow}+\hat{B}^{\dagger} \hat{c}_{\uparrow} \hat{x}_{\downarrow}+\hat{x}_{\uparrow}^{\dagger} \hat{c}_{\downarrow}^{\dagger} \hat{B}+\hat{c}_{\uparrow}^{\dagger} \hat{x}_{\downarrow}^{\dagger} \hat{B}\right) .
\end{aligned}
$$

The last part of the Hamiltonian represents the process of the creation of a biexciton through the annihilation of an exciton and a photon having opposite spins, and vice versa. The constant $g_{\Omega}$ is the radiative coupling constant of biexciton. Based on the Hamiltonian, the equation of motion of exciton, photon, and biexciton, respectively, follow

$$
\begin{aligned}
i \hbar \dot{\psi}_{x, \uparrow}= & \left(\epsilon_{x}-i \frac{\gamma_{x}}{2}\right) \psi_{x, \uparrow}+\Omega \psi_{c, \uparrow}+2 g_{++}\left|\psi_{x, \uparrow}\right|^{2} \psi_{x, \uparrow} \\
& +g_{+-}\left|\psi_{x, \downarrow}\right|^{2} \psi_{x, \uparrow}+g_{\Omega} \psi_{B} \psi_{c, \downarrow}^{*}, \\
i \hbar \dot{\psi}_{c, \uparrow}= & \left(\epsilon_{c}-i \frac{\gamma_{c}}{2}\right) \psi_{c, \uparrow}+\Omega \psi_{x, \uparrow}+g_{\Omega} \psi_{B} \psi_{x, \downarrow}^{*}-F, \\
i \hbar \dot{\psi}_{B}= & \left(\epsilon_{B}-i \frac{\gamma_{B}}{2}\right) \psi_{B}+g_{\Omega}\left(\psi_{x, \uparrow} \psi_{c, \downarrow}+\psi_{c, \uparrow} \psi_{x, \downarrow}\right) .
\end{aligned}
$$

Since in the co-circular polarization configuration the biexciton is not involved, we consider only counter-circular polarization configuration. Defining the vector of spin-down probe wave functions as $\vec{u}=\left(\psi_{x \downarrow}^{p r}, \psi_{c \downarrow}^{p r}, \psi_{B}\right)$, the dynamics of the probe wave functions is given by $i \hbar \dot{\vec{u}}=M_{\Omega}^{+-} \vec{u}-\vec{F}^{p r}$. The matrix $M_{\Omega}^{+-}$is

$$
M_{\Omega}^{+-}=\left(\begin{array}{ccc}
\epsilon_{x}+g_{+-}\left|\psi_{x \uparrow}^{p u}\right|^{2}-i \gamma_{x} / 2 & \Omega & g_{\Omega} \psi_{c \uparrow}^{p u *} \\
\Omega & \epsilon_{c}-i \gamma_{c} / 2 & g_{\Omega} \psi_{x \uparrow}^{p u *} \\
g_{\Omega} \psi_{c \uparrow}^{p u} & g_{\Omega} \psi_{x \uparrow}^{p u} & \epsilon_{B}-i \gamma_{B} / 2
\end{array}\right) .
$$



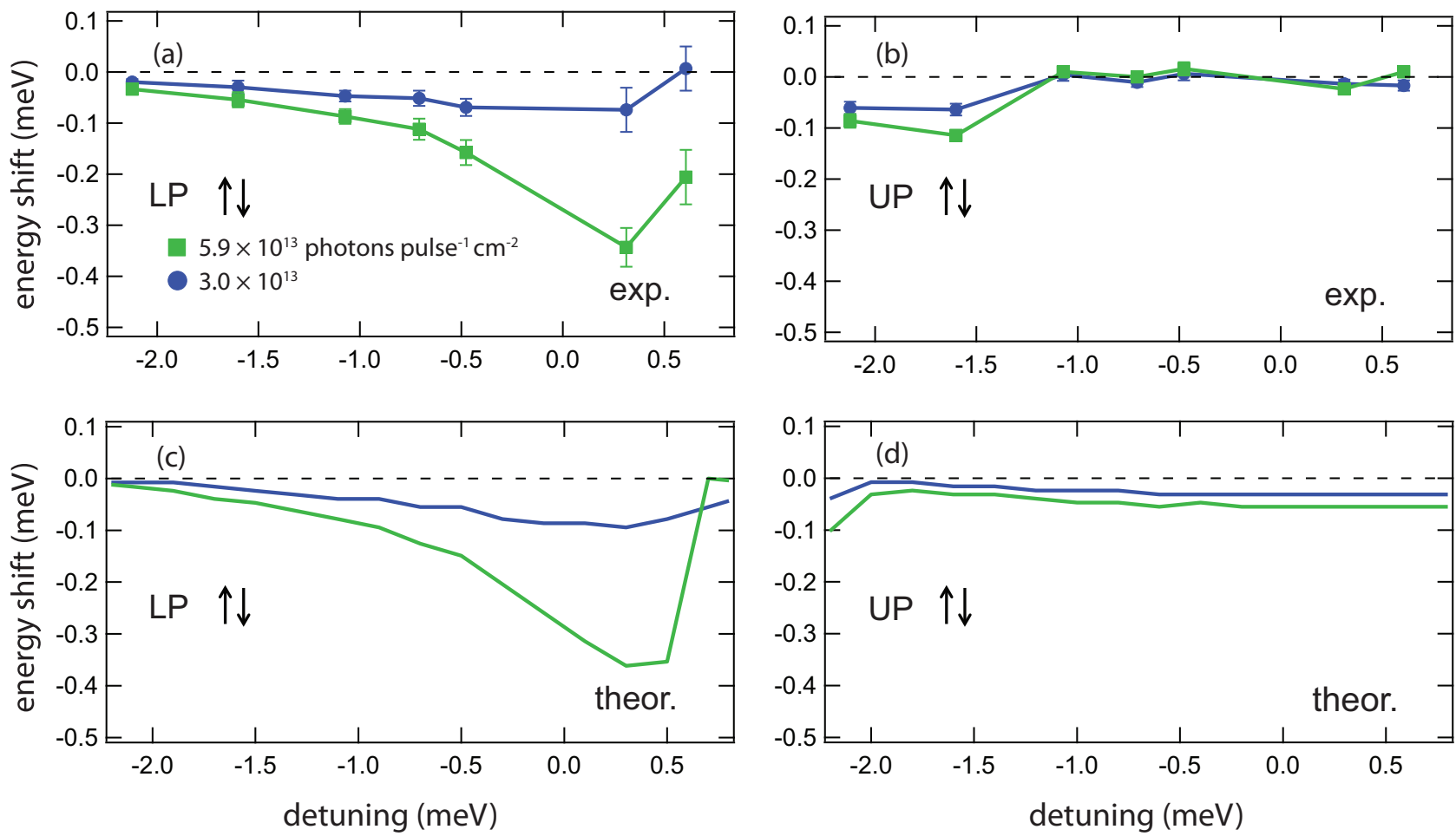

FIG. 7. (Color online) Same as Fig. 5 but considering the giant oscillator strength model as the biexciton creation process.

Compared with the bipolariton model, the photonic component of polariton plays a role in the biexciton creation process. Similarly to the main text, we try to find the value of constants $g_{+-}$and $g_{\Omega}$ that reproduces the experimental results while fixing the other parameters as in the main text. The comparison between the simulation and experiment is shown in Fig. 7. We can reproduce the lower and the upper polariton energy shift with $g_{++}: g_{+-}: g_{\Omega} \simeq 1 / n_{0}:-0.65 / n_{0}: 0.65 / \sqrt{n_{0}}(\mathrm{meV})$.

\section{Lower-polariton basis}

Following the discussion of the main text, we derive the lower-polariton interaction constant based on the giant oscillator strength model using the estimated constants. The Hamiltonian is rewritten in lower-polariton basis as

$$
\begin{aligned}
\hat{H}= & \epsilon_{c} \hat{c}_{\uparrow}^{\dagger} \hat{c}_{\uparrow}+\epsilon_{x} \hat{x}_{\uparrow}^{\dagger} \hat{x}_{\uparrow}+\Omega\left(\hat{c}_{\uparrow}^{\dagger} \hat{x}_{\uparrow}+\hat{x}_{\uparrow}^{\dagger} \hat{c}_{\uparrow}\right)+\hat{H}_{\mathrm{int}}, \\
\simeq & \epsilon_{l p} \hat{p}_{l p}^{\dagger} \hat{p}_{l p}+g_{++}|X|^{4} \hat{p}_{l p, \uparrow}^{\dagger} \hat{p}_{l p, \uparrow}^{\dagger} \hat{p}_{l p, \uparrow} \hat{p}_{l p, \uparrow} \\
& +g_{+-}|X|^{4} \hat{p}_{l p, \uparrow}^{\dagger} \hat{p}_{l p, \downarrow}^{\dagger} \hat{p}_{l p, \downarrow} \hat{p}_{l p, \uparrow} \\
& +2 g_{\Omega}\left(C X \hat{B}^{\dagger} \hat{p}_{l p, \uparrow} \hat{p}_{l p, \downarrow}+C^{*} X^{*} \hat{p}_{l p, \uparrow}^{\dagger} \hat{p}_{l p, \downarrow}^{\dagger} \hat{B}\right) .
\end{aligned}
$$

Defining the vector of the probe lower polariton wave functions as $\vec{u}=\left(\psi_{l p \downarrow}^{p r}, \psi_{B}\right)$, the dynamics follows $i \hbar \dot{\vec{u}}=M \vec{u}-\vec{F}^{p r}$, where $\vec{F}^{p r}$ is a source term. The matrix $M$ is given by

$$
M_{\Omega}=\left(\begin{array}{cc}
\epsilon_{l p}+g_{+-}|X|^{4}\left|\psi_{l p \uparrow}^{p u}\right|^{2}-i \gamma_{l p} / 2 & 2 g_{b x} C^{*} X^{*} \psi_{l p \uparrow}^{p u *} \\
2 g_{b x} C X \psi_{l p \uparrow}^{p u} & \epsilon_{B}-i \gamma_{B} / 2
\end{array}\right) .
$$

We notice that in the lower-polariton basis there are no qualitative differences between the two models [Eqs. (16) and (A5)] except for the Hopfield coefficient in front of the lowerpolariton and biexciton coupling term. The analytical solution
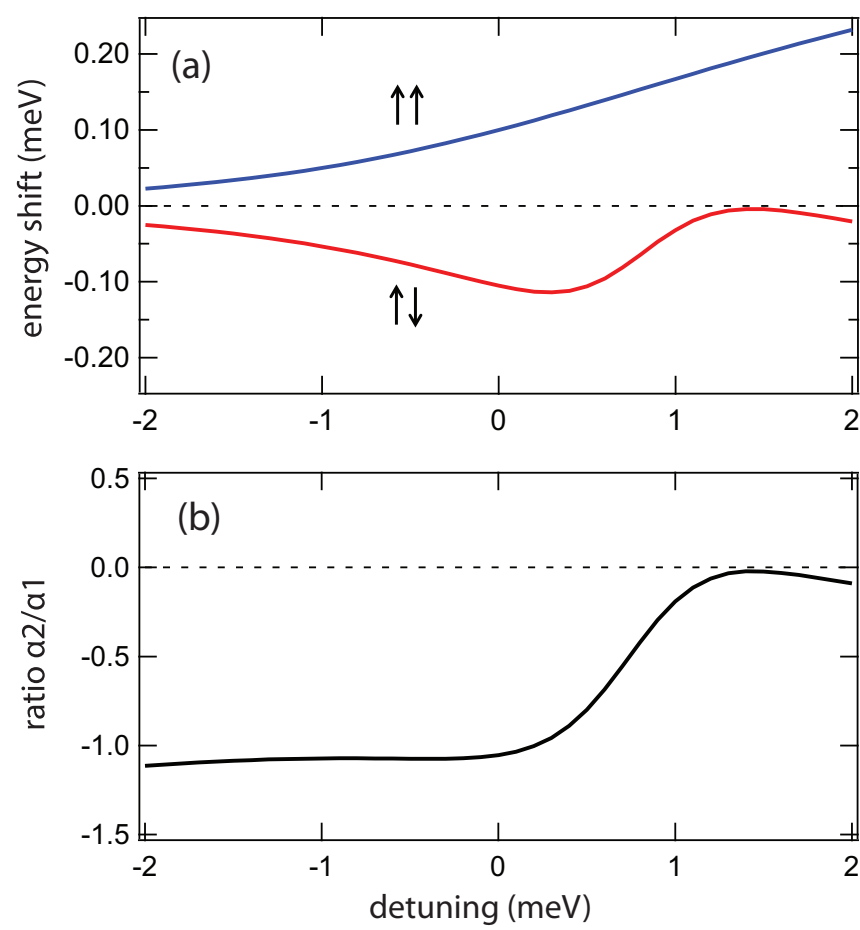

FIG. 8. (Color online) Same as Fig. 6 but considering the giant oscillator strength model as the biexciton creation process. 
of $\psi_{l p \downarrow}^{p r}(\epsilon)$ is

$$
\begin{aligned}
\psi_{l p \downarrow}^{p r}(\epsilon)= & {\left[\epsilon_{l p}-\epsilon+g_{+-}|X|^{4}\left|\psi_{l p \uparrow}^{p u}\right|^{2}-i \gamma_{l p} / 2\right.} \\
& \left.-\frac{4 g_{\Omega}^{2}|C|^{2}|X|^{2}\left|\psi_{l p \uparrow}^{p u}\right|^{2}}{\epsilon_{B}-\epsilon_{l p}-\epsilon-i \gamma_{B} / 2}\right]^{-1}
\end{aligned}
$$

The approximated interaction constant $\alpha_{2}$ becomes

$$
\alpha_{2} \simeq g_{+-}|X|^{4}-\frac{4 g_{\Omega}^{2}|C|^{2}|X|^{2}\left(\epsilon_{B}-2 \epsilon_{l p}\right)}{\left(\epsilon_{B}-2 \epsilon_{l p}\right)^{2}+\left(\gamma_{B} / 2\right)^{2}} .
$$

We show the energy shifts [Fig. 8(a)] and the ratio $\alpha_{2} / \alpha_{1}$ [Fig. 8(b)] based on Eqs. (15) and (A7). While the polaritonbiexciton coupling evolves as $|X|^{4}$ in the bipolariton model, the giant oscillator strength model has $|C|^{2}|X|^{2}$ detuning dependency. Therefore, in the giant oscillator strength model, the enhancement of the $\alpha_{2}$ interaction constant due to biexciton is weaker than that in the bipolariton model.

In this appendix, we have shown that the giant oscillator strength model also reproduces the experimental results, and both models give qualitatively similar results. Actually, due to the qualitative similarity of the two models, it is difficult to determine which model is appropriate based on these experiments. We employed the bipolariton model to describe our results based on our previous study on polariton Feshbach resonance [39] in which the resonant absorption could only be well reproduced with this model. Finally, we would like to comment on the possibility of the coexistence of two types of couplings. If there are two coupling mechanisms, the different coupling pathways constructively or destructively interfere depending on the relative phase factor between them. Even with an appropriate phase the resonant absorption could not be well reproduced [39]. Moreover, the appearance of the phase degree of freedom makes the estimation of the interaction constant impossible. Therefore, we did not consider the simultaneous existence of the two models.
[1] C. Weisbuch, M. Nishioka, A. Ishikawa, and Y. Arakawa, Phys. Rev. Lett. 69, 3314 (1992).

[2] P. G. Savvidis, J. J. Baumberg, R. M. Stevenson, M. S. Skolnick, D. M. Whittaker, and J. S. Roberts, Phys. Rev. Lett. 84, 1547 (2000).

[3] G. Messin, J. P. Karr, A. Baas, G. Khitrova, R. Houdré, R. P. Stanley, U. Oesterle, and E. Giacobino, Phys. Rev. Lett. 87, 127403 (2001).

[4] M. Saba, C. Ciuti, J. Bloch, V. Thierry-Mieg, R. André, Le Si Dang, S. Kundermann, A. Mura, G. Bongiovanni, J. L. Staehli, and B. Deveaud, Nature (London) 414, 731 (2001).

[5] S. Kundermann, M. Saba, C. Ciuti, T. Guillet, U. Oesterle, J. Staehli, and B. Deveaud, Phys. Rev. Lett. 91, 107402 (2003).

[6] W. Langbein, Phys. Rev. B 70, 205301 (2004).

[7] A. Amo, J. Lefrère, S. Pigeon, C. Adrados, C. Ciuti, I. Carusotto, R. Houdré, E. Giacobino, and A. Bramati, Nat. Phys. 5, 805 (2009).

[8] S. Utsunomiya, L. Tian, G. Roumpos, C. Lai, N. Kumada, T. Fujisawa, M. Kuwata-Gonokami, A. Löffler, S. Höfling, A. Forchel, and Y. Yamamoto, Nat. Phys. 4, 700 (2008).

[9] V. Kohnle, Y. Léger, M. Wouters, M. Richard, M. T. PortellaOberli, and B. Deveaud-Plédran, Phys. Rev. Lett. 106, 255302 (2011).

[10] V. Kohnle, Y. Leger, M. Wouters, M. Richard, M. T. PortellaOberli, and B. Deveaud, Phys. Rev. B 86, 064508 (2012).

[11] J. Kasprzak, M. Richard, S. Kundermann, A. Baas, P. Jeambrun, J. Keeling, F. Marchetti, M. H. Szymanska, R. André, J. L. Staehli, V. Savona, P. B. Littlewood, B. Deveaud, and L. S. Dang, Nature (London) 443, 409 (2006).

[12] P. Lagoudakis, P. Savvidis, J. Baumberg, D. Whittaker, P. Eastham, M. Skolnick, and J. Roberts, Phys. Rev. B 65, 161310 (2002).

[13] A. Kavokin, P. G. Lagoudakis, G. Malpuech, and J. J. Baumberg, Phys. Rev. B 67, 195321 (2003).

[14] W. Langbein, I. Shelykh, D. Solnyshkov, G. Malpuech, Y. Rubo, and A. Kavokin, Phys. Rev. B 75, 075323 (2007).
[15] A. Kavokin, G. Malpuech, and M. Glazov, Phys. Rev. Lett. 95, 136601 (2005).

[16] C. Leyder, M. Romanelli, J. P. Karr, E. Giacobino, T. Liew, M. Glazov, A. Kavokin, G. Malpuech, and A. Bramati, Nat. Phys. 3, 628 (2007).

[17] A. Amo, T. C. H. Liew, C. Adrados, E. Giacobino, A. V Kavokin, and A. Bramati, Phys. Rev. B 80, 165325 (2009).

[18] T. C. H. Liew, A. V. Kavokin, and I. A. Shelykh, Phys. Rev. B 75, 241301 (2007).

[19] T. C. H. Liew, Y. G. Rubo, and A. V. Kavokin, Phys. Rev. Lett. 101, 187401 (2008).

[20] Y. G. Rubo, Phys. Rev. Lett. 99, 106401 (2007).

[21] K. G. Lagoudakis, T. Ostatnicky, A. V. Kavokin, Y. G. Rubo, R. André, and B. Deveaud-Plédran, Science 326, 974 (2009).

[22] J. J. Baumberg, A. V. Kavokin, S. Christopoulos, A. J. D. Grundy, R. Butté, G. Christmann, D. D. Solnyshkov, G. Malpuech, G. Baldassarri Höger von Högersthal, E. Feltin, J.-F. Carlin, and N. Grandjean, Phys. Rev. Lett. 101, 136409 (2008).

[23] A. Baas, J. P. Karr, H. Eleuch, and E. Giacobino, Phys. Rev. A 69, 023809 (2004).

[24] N. A. Gippius, I. A. Shelykh, D. D. Solnyshkov, S. S. Gavrilov, Y. G. Rubo, A. V. Kavokin, S. G. Tikhodeev, and G. Malpuech, Phys. Rev. Lett. 98, 236401 (2007).

[25] T. Paraiso, M. Wouters, Y. Léger, F. Morier-Genoud, and B. Deveaud-Plédran, Nat. Mater. 9, 655 (2010).

[26] M. Wouters, T. K. Paraiso, Y. Léger, R. Cerna, F. MorierGenoud, M. T. Portella-Oberli, and B. Deveaud-Plédran, Phys. Rev. B 87, 045303 (2013).

[27] A. Amo, T. Liew, C. Adrados, R. Houdré, E. Giacobino, A. Kavokin, and A. Bramati, Nat. Photon. 4, 361 (2010).

[28] R. Cerna, Y. Léger, T. K. Paraïso, M. Wouters, F. MorierGenoud, M. T. Portella-Oberli, and B. Deveaud, Nat. Commun. 4, 2008 (2013).

[29] M. Kuwata-Gonokami, S. Inouye, H. Suzuura, M. Shirane, R Shimano, T. Someya, and H. Sakaki, Phys. Rev. Lett. 79, 1341 (1997). 
[30] K. Kavokin, P. Renucci, T. Amand, X. Marie, P. Senellart, J. Bloch, and B. Sermage, Phys. Status Solidi C 2, 763 (2005).

[31] M. Vladimirova, S. Cronenberger, D. Scalbert, K. V. Kavokin, A. Miard, A. Lemaître, J. Bloch, D. Solnyshkov, G. Malpuech, and A. V. Kavokin, Phys. Rev. B 82, 075301 (2010).

[32] L. Ferrier, E. Wertz, R. Johne, D. D. Solnyshkov, P. Senellart, I. Sagnes, A. Lemaître, G. Malpuech, and J. Bloch, Phys. Rev. Lett. 106, 126401 (2011).

[33] N. H. Kwong, R. Takayama, I. Rumyantsev, M. KuwataGonokami, and R. Binder, Phys. Rev. B 64, 045316 (2001).

[34] M. Wouters, Phys. Rev. B 76, 045319 (2007).

[35] W. Casteels and M. Wouters, Phys. Rev. A 90, 043602 (2014).

[36] C. Ciuti, P. Schwendimann, B. Deveaud, and A. Quattropani, Phys. Rev. B 62, R4825 (2000).

[37] R. Stanley, R. Houdre, U. Oesterle, M. Gailhanou, and M. Ilegems, Appl. Phys. Lett. 65, 1883 (1994).

[38] K. L. Hall, G. Lenz, E. P. Ippen, and G. Raybon, Opt. Lett. 17, 874 (1992).

[39] N. Takemura, S. Trebaol, M. Wouters, M. T. Portella-Oberli, and B. Deveaud, Nat. Phys. 10, 500 (2014).

[40] A. L. Ivanov, M. Hasuo, N. Nagasawa, and H. Haug, Phys. Rev. B 52, 11017 (1995).

[41] E. Hanamura, Solid State Commun. 12, 951 (1973).

[42] I. Carusotto, T. Volz, and A. Imamoğlu, Europhys. Lett. 90, 37001 (2010).
[43] C. Ciuti, V. Savona, C. Piermarocchi, A. Quattropani, and P. Schwendimann, Phys. Rev. B 58, 7926 (1998).

[44] V. Axt, K. Victor, and T. Kuhn, Phys. Status Solidi B 206, 189 (1998).

[45] N. H. Kwong, R. Takayama, I. Rumyantsev, M. KuwataGonokami, and R. Binder, Phys. Rev. Lett. 87, 027402 (2001).

[46] R. Takayama, N. Kwong, I. Rumyantsev, M. Kuwata-Gonokami, and R. Binder, Eur. Phys. J. B 25, 445 (2002).

[47] T. Östreich, K. Schönhammer, and L. J. Sham, Phys. Rev. Lett. 74, 4698 (1995).

[48] M. Grassi Alessi, F. Fragano, A. Patanè, M. Capizzi, E. Runge, and R. Zimmermann, Phys. Rev. B 61, 10985 (2000).

[49] M. Kuwata-Gonokami, T. Aoki, C. Ramkumar, R. Shimano, and Y. Svirko, J. Lumin. 87, 162 (2000).

[50] A. L. Ivanov and H. Haug, Phys. Rev. B 48, 1490 (1993).

[51] T. Lecomte, D. Taj, A. Lemaitre, J. Bloch, C. Delalande, J. Tignon, and P. Roussignol, Phys. Rev. B 89, 155308 (2014)

[52] H. H. Yaffe, Y. Prior, J. P. Harbison, and L. T. Florez, J. Opt. Soc. Am. B 10, 578 (1993).

[53] S. Inouye, M. R. Andrews, J. Stenger, H.-J. Miesner, D. M. Stamper-Kurn, and W. Ketterle, Nature (London) 392, 151 (1998).

[54] E. Timmermans, P. Tommasini, R. Côté, M. Hussein, and A. Kerman, Phys. Rev. Lett. 83, 2691 (1999).

[55] M. Theis, G. Thalhammer, K. Winkler, M. Hellwig, G. Ruff, R. Grimm, and J. H. Denschlag, Phys. Rev. Lett. 93, 123001 (2004). 